Document downloaded from:

http://hdl.handle.net/10251/55107

This paper must be cited as:

Muller, AA.; Soto Pacheco, P.; Dascalu, D.; Neculoiu, D.; Boria Esbert, VE. (2011). A 3D Smith Chart based on the Riemann Sphere for Active and Passive Microwave Circuits. IEEE Microwave and Wireless Components Letters. 21(6):286-288.

doi:10.1109/LMWC.2011.2132697.

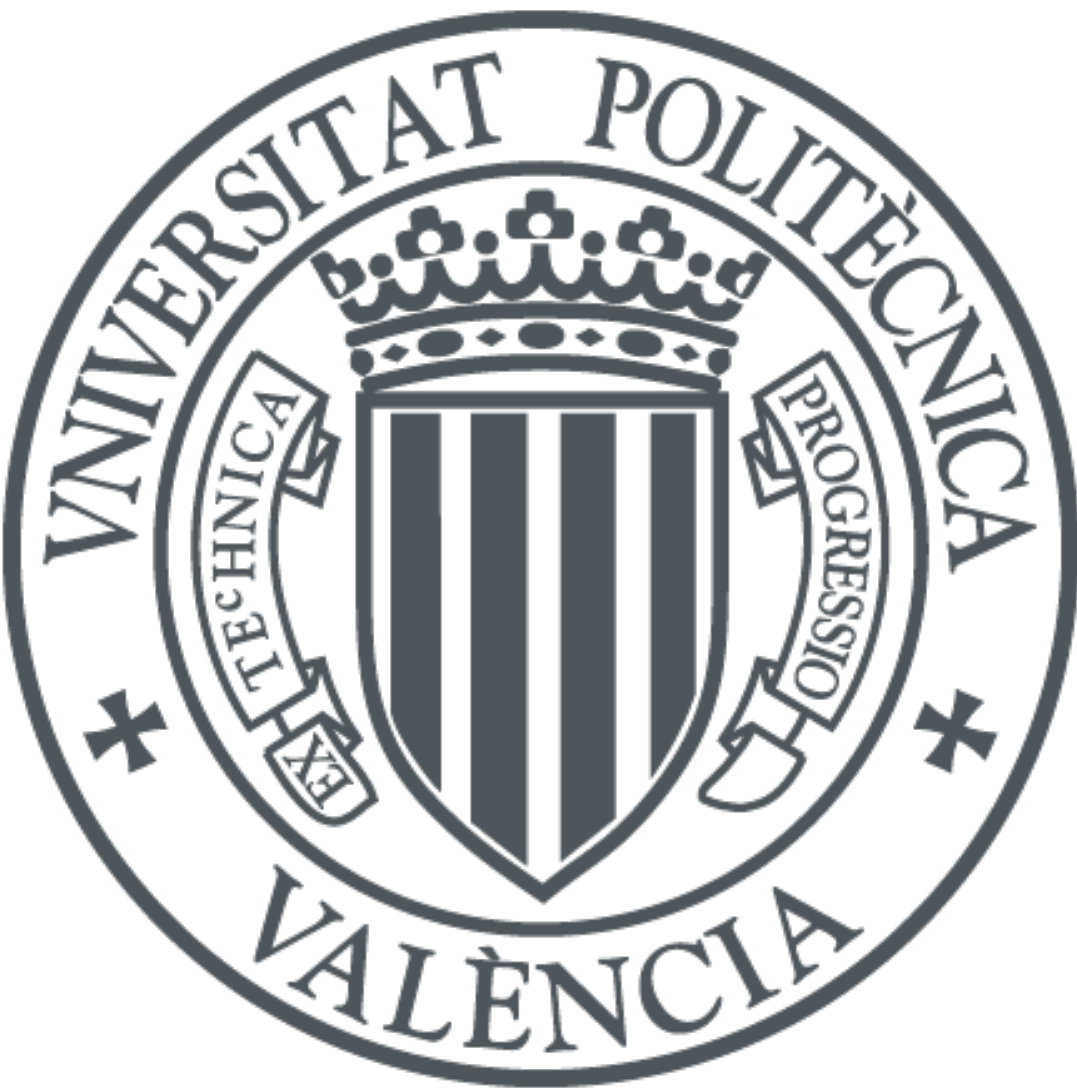

The final publication is available at

http://dx.doi.org/10.1109/LMWC.2011.2132697

Copyright Institute of Electrical and Electronics Engineers (IEEE)

Additional Information

"(C) (C) 20xx IEEE. Personal use of this material is permitted. Permission from IEEE must be obtained for all other uses, in any current or future media, including reprinting/republishing this material for advertising or promotional purposes, creating new collective works, for resale or redistribution to servers or lists, or reuse of any copyrighted component of this work in other works." 


\title{
A 3D Smith Chart based on the Riemann Sphere for Active and Passive Microwave Circuits
}

\author{
Andrei A. Muller, Student Member, IEEE, Pablo Soto, Member, IEEE, Dan Dascalu, Senior Member, IEEE, \\ Dan Neculoiu, Member, IEEE, and Vicente E. Boria, Senior Member, IEEE
}

\begin{abstract}
This paper proposes a spherical 3D Smith Chart suitable for representing both active and passive microwave circuits. Using the mathematical concept of the Riemann sphere, the extended reflection coefficient plane is transformed into the surface of the unit sphere. Since the proposed Smith Chart compiles the whole complex plane, all possible loads are included. A simple graphic tool is thus obtained that successfully unifies active and passive circuits. In addition, lossy lines with complex characteristic impedances can also be represented. The letter presents the 3D Smith Chart, provides its main governing equations, and also enumerates its more important properties.
\end{abstract}

Index Terms - Smith Chart, transmission lines, immittance, reflection coefficient, active circuits, lossy circuits.

\section{INTRODUCTION}

$\mathrm{O}$ NE of the more useful and widely known microwave tools is the Smith Chart [1]. This graphic chart proposed in the late 30s has survived the advent of personal computers and computer aided design (CAD) tools, since it provides an excellent visual insight of microwave problems. Its simplicity and beauty are inherited from the mathematical properties of Möbius transformations, also related to geometrical art [2].

To have a finite and practical size, the Smith Chart is constrained to the unit circle. Hence, loads with reflection coefficient magnitude greater than 1 cannot be plotted. These loads often appear in active circuits [3] and in lossy transmission lines with complex characteristic impedances [4].

A spherical 3D Smith Chart has been recently proposed to overcome these limitations [5]. The heuristic approach in [5], however, does not include mathematical support necessary to move from the conceptual idea to a practical tool. Although a new theory has been reported [6], the resulting representation

Manuscript received January 3, 2011. The stage of A.A. Muller at Universitat Politècnica de València (UPV) has been carried out in the frame of the POSDRU ID 7713 European Project. This work has been partly supported by Ministerio de Ciencia e Innovación, Spanish Government, under Research Project TEC2010-21520-C04-01.

A.A Muller, D. Dascalu and Dan Neculoiu are with the Romanian National Institute for R\&D in Microtechnologies (IMT), 38610, Bucharest, Romania (phone: +40-21-269-0770, fax: +40-021-269-0772, e-mail: andrei.stefan1@gmail.com; dan.dascalu@imt.ro; dan.neculoiu@imt.ro). D. Dascalu and D. Neculoiu are also with University "Politehnica" of Bucharest, Faculty of Electronics, Communications and Information Technology.

P. Soto and V.E. Boria are with Grupo de Aplicaciones de las Microondas (GAM), iTEAM, Universitat Politècnica de València, E-46022, Valencia, Spain (e-mail: pabsopac@dcom.upv.es; vboria@dcom.upv.es).

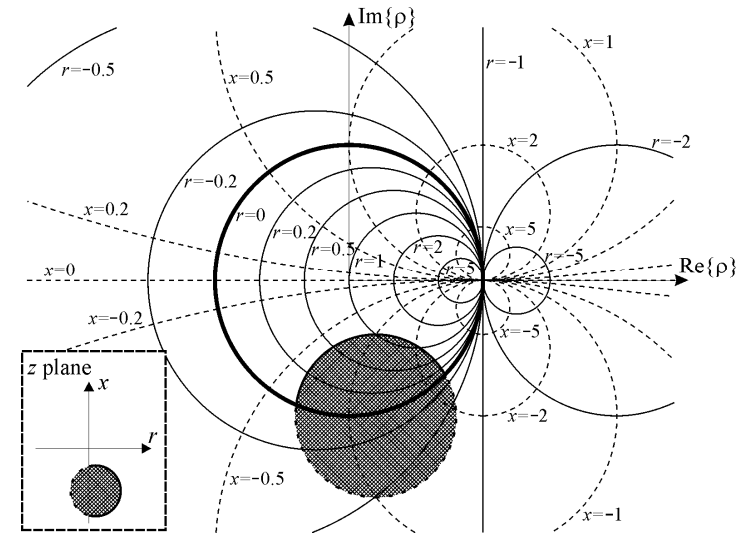

Fig. 1. Reflection coefficient plane with some transformed constant resistance (continuous lines) and reactance (dashed lines) circumferences by using (1). The Smith Chart boundary is drawn with a thick line. A shaded stability circle partly outside the unit circle is also plotted in both the $\rho$-plane and the $z$-plane.

distorts the constant resistance and reactance circumferences into ellipses in the reflection coefficient plane, and also results in complicated transforming equations, making very difficult the visual and intuitive interpretation of microwave problems.

In this letter, a new theory based on the mathematical concept of the Riemann sphere is presented. A simpler representation able to keep the shape of the planar Smith Chart is thus obtained. In addition, the resulting $3 \mathrm{D}$ chart has many practical properties than can provide a better insight into the problem and ease the task of microwave engineers.

\section{Planar Smith Chart and MöbiUs transformations}

In the extended complex plane, that includes the point at infinity $(\mathbb{C} \cup\{\infty\})$, any Möbius transformation is an inversive transformation with very interesting properties. They provide a conformal mapping, transform generalized circles (i.e., finite radius circles and infinite lines) into generalized circles, map points symmetric to a circle into points also symmetric to their image circle, and there is a unique Möbius transformation that maps any three points to any other three points [7].

The relationship between the normalized impedance $z$ and the reflection coefficient $\rho$ can be represented by means of Möbius transformations for a wide range of transmission lines [8]. This transformation, in the case of reciprocal lines of characteristic impedance $Z_{0}$, takes the known form

$$
\rho=\frac{z-1}{z+1} \quad ; \quad z=r+\mathrm{j} x=Z / Z_{0} .
$$




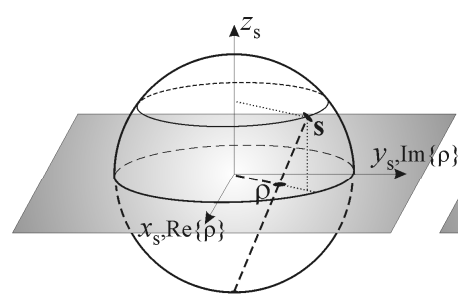

(a)

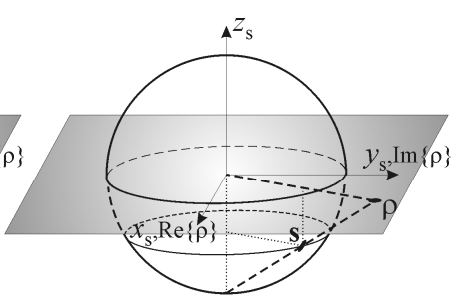

(b)
Fig. 2. Stereographic projection from the reflection coefficient plane to the unit sphere surface using the sphere south pole. Point inside the unit circle in (a) and outside in (b).

From (1) it can be concluded that impedances with positive normalized resistance $r$ map in the unit circle (with the zero resistance loads placed on its circumference), whereas loads with negative $r$ are placed outside the unit circle. It can also be proven that the $z$-plane unit circle $|z|=1$ is mapped to the $\rho$-plane imaginary axis.

Although the Möbius transformation (1) spans the whole $z$ and $\rho$ extended complex planes, the classic planar Smith Chart only considers the $|\rho| \leq 1$ region. This region contains the more common loads in practice. There are some applications, however, where loads placed outside the unit circumference can appear, such as the stability analysis and design of amplifiers and oscillators [3]. They also can arise in lossy transmission lines with complex $Z_{0}$, where even passive loads can have normalized impedances with negative real part.

Fig. 1 depicts the conformal transformation (1) in the $\rho$-plane, including some constant resistance and reactance circumferences. A stability circle partly outside the region considered in the planar Smith Chart has also been plotted. The circle points placed outside the unit circumference corresponds to negative resistance loads in the normalized impedance $z$-plane.

\section{3D Spherical Smith Chart AND the RiEmanN SPHERE}

The extended complex $\rho$-plane includes all the passive and active loads, but it is difficult to represent in practice because includes the point at infinity. Fortunately, Riemann interpreted the numbers in the extended complex plane as points on a sphere. Using Ptolemy's theory on celestial spheres, Riemann applied a stereographic projection to perform a one-to-one correspondence between the extended complex plane and the unity radius sphere (i.e., the unit sphere).

The spherical Smith Chart proposed in this paper is created following these principles, since a stereographic projection of the reflection coefficient plane located in the equatorial plane is performed by using the sphere south pole. According to this projection, the points in the reflection coefficient plane lying inside the unit circle are mapped to the northern hemisphere (see Fig. 2a). Conversely, the points placed outside this circle are moved to the southern hemisphere (see Fig. 2b). Finally, the unit circumference points are mapped to themselves.

This procedure provides a unit Riemann sphere, whose surface can be considered as a 3D Smith Chart which includes all complex loads. From geometrical considerations, it can be

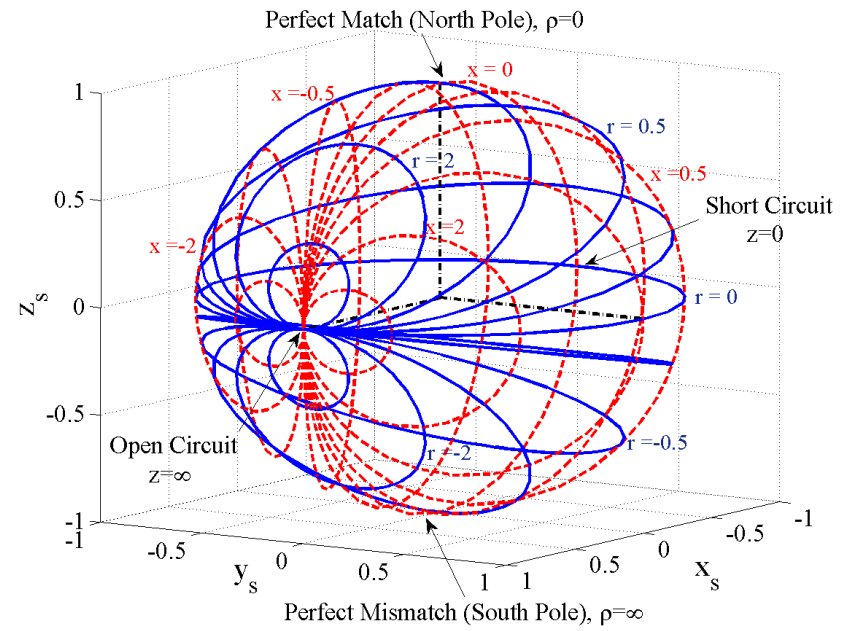

Fig. 3. 3D spherical Smith Chart obtained after performing the stereographic projection. The same constant normalized resistance (continuous lines) and reactance (dashed lines) circles plotted in Fig. 1 are shown, which are alternatively labeled. The dash-dotted line represents the sphere unitary axes.

inferred that a generic $\rho$-plane point

$$
\rho=\rho_{\mathrm{r}}+\mathrm{j} \rho_{\mathrm{i}}=|\rho| e^{\mathrm{j} \varphi_{\rho}}
$$

is mapped into the surface point of the unit sphere given by

$$
\mathbf{s}\left(\rho=\rho_{\mathrm{r}}+\mathrm{j} \rho_{\mathrm{i}}\right)=\left(\frac{2 \rho_{\mathrm{r}}}{1+|\rho|^{2}}, \frac{2 \rho_{\mathrm{i}}}{1+|\rho|^{2}}, \frac{1-|\rho|^{2}}{1+|\rho|^{2}}\right)
$$

which can also be expressed in terms of the load normalized resistance $r$ and reactance $x$ in the form

$$
\mathbf{s}(z=r+j x)=\frac{1}{|z|^{2}+1}\left(|z|^{2}-1,2 x, 2 r\right)
$$

Conversely, a given point $\mathbf{s}=\left(x_{\mathrm{s}}, y_{\mathrm{s}}, z_{\mathrm{s}}\right)$ in the surface of the unit sphere (i.e., satisfying $x_{\mathrm{s}}^{2}+y_{\mathrm{s}}^{2}+z_{\mathrm{s}}^{2}=1$ ) is mapped to

$\rho(\mathbf{s})=\rho_{\mathrm{r}}(\mathbf{s})+\mathrm{j} \rho_{\mathrm{i}}(\mathbf{s})=\frac{(r-1)^{2}+x^{2}+2 \mathrm{j} x}{(r+1)^{2}+x^{2}}=\frac{x_{s}+\mathrm{j} y_{s}}{1+z_{s}}$

in the $\rho$-plane (i.e., the sphere equatorial plane).

After performing such a mapping of the extended complex $\rho$-plane, the spherical Smith Chart in Fig. 3 is finally obtained. The sphere north pole represents the perfect match point; whereas the normalized impedance $z=-1$ is located in the south pole, being the stereographic projection of the infinite reflection coefficient or perfect mismatch. The open circuit is the sphere surface point on the positive $x_{\mathrm{s}}(\operatorname{or} \operatorname{Re}\{\rho\})$ axis whereas the short circuit is placed on the negative one. Note that all constant reactance and resistance circumferences touches the equatorial plane in the open circuit point $(1,0,0)$.

From the Riemann sphere properties, it can be stated that the projection is conformal for an observer inside the sphere, 


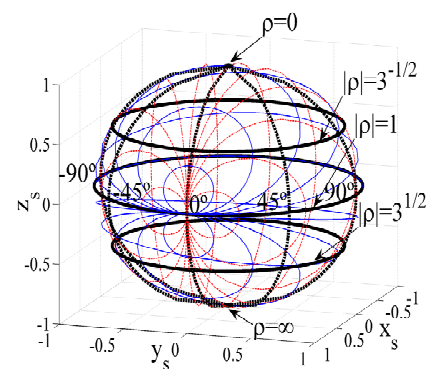

(a)

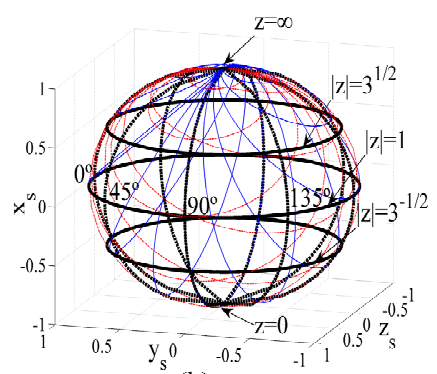

(b)
Fig. 4. Constant magnitude circumferences (continuous) and phase meridians (dashed) for the reflection coefficient in (a). Constant normalized impedance magnitude (continuous) and quality factor circumferences (dashed) in (b). The quality factor circumferences are labeled with the angle $\psi$ between its plane and the $y_{\mathrm{s}}$ axis (whose tangent is precisely the circuit quality factor).

as well as generalized circles in the $\rho$-plane are mapped into circles in the sphere surface (where infinite lines in the complex $\rho$-plane are transformed into circles passing the south pole) [9]. The sphere in Fig. 3 shows these properties.

\section{Properties of THE 3D SphericAl SMith CHART}

The 3D spherical Smith Chart in Fig. 3 has many useful properties. This section summarizes the more relevant ones.

The position of the different type of loads is considered in the first place. The upper hemisphere of the spherical Smith Chart is inhabited by the normalized impedances with positive real part, whereas the negative resistance loads are mirrored on the lower hemisphere. In the equatorial circumference, the loads are purely reactive (see Fig. 3). On the other hand, the central or Greenwich sphere meridian in the XZ plane contains the purely resistive loads. The loads on the left hemisphere $\left(y_{\mathrm{s}}<0\right)$ exhibit a normalized capacitive behaviour, whereas the inductive circuits are placed on the right one $\left(y_{\mathrm{s}}>0\right)$. Note that the four quadrants in a $2 \mathrm{D}$ cut with any plane parallel to the YZ plane contain different types of loads.

In the 3D Smith Chart, the magnitude of the reflection coefficient is only related with the $z_{\mathrm{s}}$ coordinate of the sphere (see (3)), and the phase of the reflection coefficient is equal to the angle of the point with respect to the $x_{\mathrm{s}}$ sphere axis (see Fig. 2). As a result, the sphere latitude provides the reflection coefficient magnitude and the meridian its phase according to

$$
\rho=|\rho| \mathrm{e}^{\mathrm{j} \varphi_{\rho}} \quad ; \quad|\rho|=\sqrt{\frac{1-z_{\mathrm{s}}}{1+z_{\mathrm{s}}}}, \varphi_{\rho}=\operatorname{atan}\left(\frac{y_{\mathrm{s}}}{x_{\mathrm{s}}}\right) .
$$

Fig. 4a plots some circumferences with constant reflection coefficient magnitude and phase. The reflection coefficient magnitude increases from the north pole $(\rho=0)$ to the south pole $(\rho=\infty)$, being $|\rho|=1$ in the equatorial plane. Interestingly, a movement of length $l$ on the transmission line towards generator or load is carried out, respectively, with a clockwise or anticlockwise turn of $\theta=2 \beta l$ around the sphere $z_{\mathrm{s}}$ axis, where $\beta$ is the line phase constant (in a lossless line, the turn is performed in the same latitude circumference).

Moreover, the normalized impedance complex magnitude $|z|$ and angle $\varphi_{\mathrm{z}}$ can also be easily obtained from the load coordinates in the sphere. From (4) it can be deduced that

$$
|z|=\sqrt{r^{2}+x^{2}}=\sqrt{\frac{1+x_{\mathrm{s}}}{1-x_{\mathrm{s}}}}, \cot \left(\varphi_{\mathrm{z}}\right)=Q=\frac{r}{x}=\frac{\mathrm{z}_{\mathrm{s}}}{\mathrm{y}_{\mathrm{s}}}
$$

thus constant $x_{\mathrm{s}}$ circumferences contain the points with the same normalized impedance magnitude. On the other hand, the circumference in a plane including the $x_{\mathrm{s}}$ axis and forming a given angle $\psi$ with the $y_{\mathrm{s}}$ sphere axis collects the loads with a quality factor $Q$ equal to $\tan (\psi)$ (see Fig. 4b).

It can be easily proven that the $3 \mathrm{D}$ admittance Smith Chart is obtained by altering the sign of the reflection coefficient. From a given load, this can be carried out with a $180^{\circ}$ rotation around the $z_{\mathrm{s}}$ axis (in the same latitude circumference). After this rotation, the normalized resistance $r$ and reactance $x$ circumferences convert into normalized conductance $g$ and susceptance $b$ circumferences. The equations and properties of the impedance Smith Chart also hold provided that $r$ and $x$ are replaced by $g$ and $b$, respectively, and the signs of the real and imaginary part of the reflection coefficient are changed.

\section{CONCLUSION}

This letter proposes a generalized Smith Chart based on the Riemann sphere. Using the concept of extended complex plane and inversive geometry, the generalized Smith Chart can include all the complex loads in the reflection coefficient plane. This leads to a 3D representation with simple governing equations and many useful properties, which can be a valuable graphic tool to handle microwave problems involving any type of loads.

\section{ACKNOWLEDGMENT}

Andrei A. Muller would like to thank Acad. Jose Bonet and coworkers from Instituto Universitario de Matemática Pura y Aplicada (IUMPA), at Universitat Politècnica de València, Spain, for their support in his doctoral stage, which is supervised by Acad. Dan Dascalu.

\section{REFERENCES}

[1] P. H. Smith, "Transmission-line calculator," Electronics, vol. 12, pp.2931, Jan. 1939.

[2] M. S. Gupta, "Escher's art, Smith Chart and hyperbolic geometry," IEEE Microwave, vol. 7, pp 67-76, Oct. 2006.

[3] P. D. Abrie, Design of RF and Microwave Amplifiers and Oscillators. Norwood, MA: Artech House, 2009.

[4] R. J. Vernon and S. R. Seshadri, "Reflection coefficient and reflected power on a lossy transmission line", Proc. IEEE, vol. 57, pp. 101-102, Jan. 1969

[5] C. Zelley, "A spherical representation of the Smith Chart," IEEE Microwave, vol. 8, pp. 60-66, Jun. 2007.

[6] Y. Wu, Y. Zhang, Y. Liu, and H. Huang, "Theory of the spherical generalized Smith Chart," Microwave Opt. Technol. Lett., vol 51, pp. 95-97, Jan. 2009.

[7] D. A. Brannan, M. F. Esplen, J. J. Gray, Geometry. Cambridge, UK: Cambridge University Press, 1999.

[8] Y. Wu, Y. Liu, and H. Huang, "Extremely generalized planar Smith Chart based on Möbius transformations," Microwave Opt. Technol. Lett., vol. 51, pp. 1164-1167, May 2009.

[9] T. Needham, Visual Complex Analysis. New York, NY: Oxford University Press, 1997. 